\title{
Endosalpingiosis with concurrent endometriosis of ovary masquerading as ovarian malignancy
}

\author{
Amita Shah ${ }^{1}$, Rinchen Zangmo ${ }^{2 *}$, Kalpana Jain ${ }^{3}$
}

\begin{abstract}
${ }^{1}$ Department of Obstetrics and Gynecology, Columbia Asia Hospital, Palam Vihar, Gurgaon, Haryana, India ${ }^{2}$ Department of Obstetrics and Gynecology, All India Institute of Medical Sciences New Delhi, India ${ }^{3}$ Department of Pathology and Laboratory Services, Columbia Asia Hospital, Palam Vihar, Gurgaon, Haryana, India
\end{abstract}

Received: 18 April 2019

Revised: 05 May 2020

Accepted: 11 May 2020

\section{*Correspondence:}

Dr. Rinchen Zangmo,

E-mail: rinchhen.zn@gmail.com

Copyright: ( ) the author(s), publisher and licensee Medip Academy. This is an open-access article distributed under the terms of the Creative Commons Attribution Non-Commercial License, which permits unrestricted non-commercial use, distribution, and reproduction in any medium, provided the original work is properly cited.

\begin{abstract}
Endosalpingiosis is a rare gynecological disorder of müllerian origin, characterized by the presence of tubal epithelium outside the fallopian tube, which involves structures of the female genital tract, peritoneum, and subperitoneal tissues. Endosalpingiosis can be associated with endometriosis or endocervicosis, although it often appears alone. Authors report a case of endosalpingiosis with concurrent endometriosis in a 42-year-old P2L1 patient. The patient presented to us with complaints of heaviness in lower abdomen, a feeling a lump in the lower abdomen and low-grade fever for 15 days. On per abdominal examination, a large solid cystic mass up to 20 weeks size was felt, which was more on the left side. Cervix was normal on speculum examination, the same mass was felt on per vaginal examination, separate from the uterus, the right fornix appeared free. Patient was asked to get a set of investigations done and to review as early as possible. An exploratory laparotomy with peritoneal wash cytology, total abdominal hysterectomy, bilateral salpingo-ophorectomy with supracolic and infracolic omentectomy and bilateral pelvic lymph nodes dissection was done on 18/07/18. Per operatively, there was a large cystic mass occupying the abdominal cavity adhered to the bowel and to posterior wall of the uterus, adhesiolysis followed by staging laparotomy was done. Patient's postoperative course was uneventful and she was discharged on the $5^{\text {th }}$ day of surgery in stable condition. The final histopathology report was suggestive of endosalpingiosis with concurrent endometriosis.
\end{abstract}

Keywords: Adnexal mass, Endometriosis, Endosalpingiosis, Ovarian malignancy

\section{INTRODUCTION}

Endosalpingiosis is a rare gynecological disorder of müllerian origin, also known as müllerian cyst of uterus or müllerianosis. ${ }^{1,2}$ It is characterized by the presence of tubal epithelium outside the fallopian tube, which involves structures of the female genital tract, peritoneum, and sub-peritoneal tissues. ${ }^{3}$ This condition was first reported in the literature in $1930 .{ }^{4}$ It is believed that the condition occurs due to metaplastic transformation of coelomic epithelium into tubal-like epithelium. ${ }^{2,4}$
Endosalpingiosis can be associated with endometriosis or endocervicosis, although it often appears alone. One retrospective study quoted concurrent presence of endometriosis in $34.5 \%$ patients of endosalpingiosis. ${ }^{5,6} \mathrm{~A}$ strong correlation between endosalpingiosis and gynecological malignancy in premenopausal females ( $p$ value <0.0001) with no significant correlation in postmenopausal females was also described by that study.

Authors present a case of a 42-year-old female with endosalpingiosis with concurrent endometriosis. 


\section{CASE REPORT}

A 42-year-old P2L1 patient, present to the outpatient department of this case hospital with complaints of heaviness in lower abdomen, a feeling a lump in the lower abdomen and low-grade fever for the last 15 days. Her cycles were regular with average flow and there was no history of dysmenorrhea. She had delivered two children, first was a normal delivery and the second was born by Caesarean section 12 years back. There was no history of loss of weight, no history of any bowel and bladder disturbance. On examination, her vitals were stable, she was afebrile, and chest and CVS examinations were unremarkable. On per abdominal examination, a large solid cystic mass up to 20 weeks size was felt, which was more on the left side. Cervix was normal on speculum examination, the same mass was felt on per vaginal examination, separate from the uterus, the right fornix appeared free. Patient was asked to get a set of investigations done and to review as early as possible. On follow up, all fever investigations were normal, her CA125 was 176, CEA was 2.66, Ultrasound showed a large left ovarian complex cystic lesion $(16 \times 15 \times 15 \mathrm{~cm})$ in the left ovary with exophytic solid component and no free fluid. A PET scan was also done, which was suggestive of a large complex cystic malignant left ovarian mass of about $10.8 \times 14 \times 18.5 \mathrm{~cm}$ extending up to umbilicus compressing distal third of left ureter causing mid hydronephrosis with enlarged retroperitoneal lymph nodes (Figure 1).
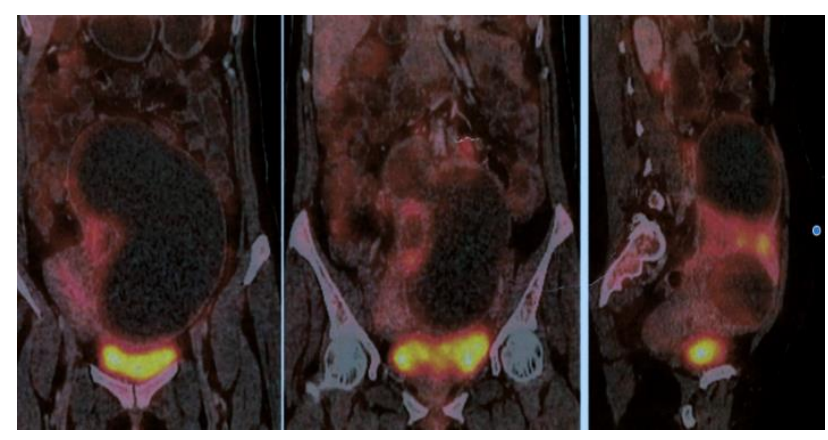

Figure 1: PET scan findings: large, complex, cystic, malignant appearing mass measuring $10.8 \times 14.0 \times 18.5$ cm extending into lower abdomen.

The patient was found to have moderate anemia during work up, for which 3 units of packed red cells were transfused before she was taken up for surgery. An exploratory laparotomy with peritoneal wash cytology, total abdominal hysterectomy, bilateral salpingoophorectomy with supracolic and infracolic omentectomy and bilateral pelvic lymph nodes dissection was done on $18^{\text {th }}$ July 2018 . Per operatively, there was a large cystic mass occupying the abdominal cavity adhered to the bowel and to posterior wall of the uterus, adhesiolysis followed by staging laparotomy was done.

Patient's postoperative course was uneventful and she was discharged on the $5^{\text {th }}$ day of surgery in stable condition. The final histopathology report was suggestive of endosalpingiosis with concurrent endometriosis (Figure 2).

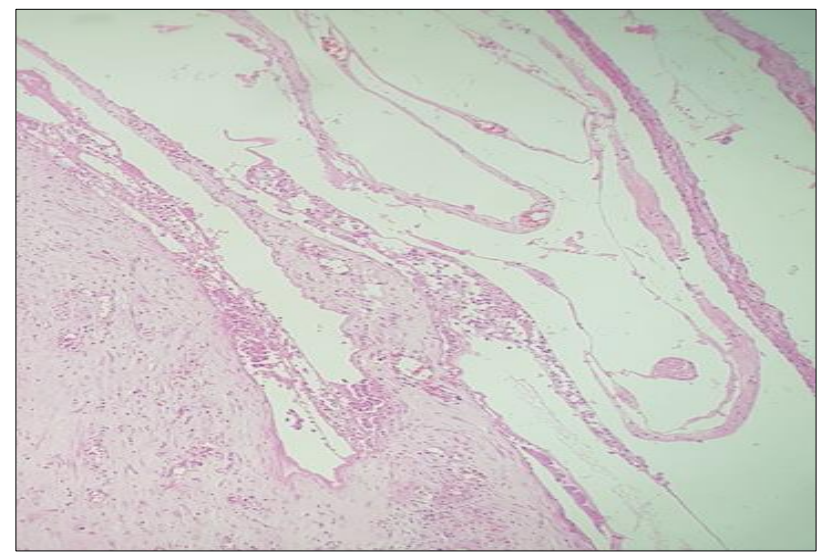

Figure 2: Multiple cystic spaces lined by flat, cuboidal to ciliated columnar epithelium, suggestive of endosalpingiosis with concurrent endometriosis.

Patient came for follow up on $15^{\text {th }}$ April 2019, she was free of symptoms. Her abdominal and vaginal examinations were within normal limits apart from the healed surgical scar.

\section{DISCUSSION}

Endosalpingiosis can be diagnosed incidentally during work-up of other conditions such as infertility, chronic pelvic pain, pelvic mass and menstrual irregularity.

Prentice et al, observed that $59.1 \%$ patients with endosalpingiosis had previous gynecologic and abdominal surgery, and a history of tubal disease was documented in $33.6 \%$ of cases, which raises the possibility that peritoneal implantation may be a factor in the etiology of endosalpingiosis. ${ }^{5}$ The patient in this study too, had previous 1 caesarean section.

Gross appearance of endosalpingiosis may mimic a primary peritoneal tumor or papillary carcinoma of ovary, but the absence of mitotic activity or atypia on histopathology contradicts the diagnosis of carcinoma. 6 Extensive endosalpingiosis involving the vaginal cuff, pelvic peritoneum, anterior abdominal wall and serosa of the sigmoid colon has been reported in a 68-year-old postmenopausal woman who was operated for Stage 1A carcinoma of ovary in the past.

An extensive surgery with upper vaginectomy, complete pelvic peritoniectomy, excision of the bladder peritoneum and the lower anterior abdominal wall peritoneum and cystic lesions of the sigmoid and infracolic omentectomy was also performed based on patient's previous history of ovarian cancer and the per op findings which were very much in favor of recurrence of ovarian cancer, but the histopathological report showed endosalpingiosis. ${ }^{7}$ 
Asymptomatic endosalpingiosis does not require any treatment. It may become symptomatic by mechanical irritation of abdominal organs and in such cases; surgical removal of the cystic structures may effectively abolish the symptoms.

Clement and young reported four cases of florid cystic endosalpingiosis of the uterus n 1999, of which, one had transmural uterine involvement by endosalpingiosis, which was the first reported case of transmural uterine endosalpingiosis. ${ }^{8}$ Hemalatha et al, report a case wherein a patient had presented with third degree uterine prolapse along with cystocoele, rectocoele and enterocoele for which a vaginal hysterectomy was performed. A cyst attached to the serosal surface of uterine fundus was incidentally detected per operatively. Bilateral fallopian tubes and ovaries were separate and normal in appearance, there was no continuity between the cyst and the endometrial cavity. Microscopic examination demonstrated presence of fallopian tube-like epithelium in the parametrium and the cyst wall lined by the same type of epithelium, which led to the diagnosis of cystic endosalpingiosis of uterus. ${ }^{9}$ Santeusanio $G$ et al have reported a case of omental endosalpingiosis. ${ }^{10}$

\section{CONCLUSION}

Few studies have described association of endosalpingiosis with epithelial ovarian cancer, thus, although endosalpingiosis in itself is not a malignant condition, it is advisable to keep the patients under follow up after surgery, specially the patients who have undergone conservative surgery.

\section{ACKNOWLEDGMENTS}

Authors would like to thank patient for having given us the permission to report her case in a medical journal for the benefit of doctors from this fraternity.

Funding: No funding sources Conflict of interest: None declared Ethical approval: Not required

\section{REFERENCES}

1. Lui MW, Ngu SF. Mullerian cyst of the uterus misdiagnosed as ovarian cyst on pelvic sonography. J Clin Ultrasound. 2014;42(3):183-84.

2. Bermejo R, Gomez A. Peritoneal mullerian tumorlike (endosalpingiosis leiomyomatosis peritoneal): a hardly known entity. Case Rep Obstet Gynecol. 2012;2012:329416.

3. Singh N, Murali S. Florid cystic endosalpingiosis, masquerading as malignancy in a young patient: a brief review. BMJ Case Rep. 2014:bcr201320164

4. Sampson JA. Post-salpingectomy endometriosis, (endosalpingiosis). Am J Obstet Gynecol. 1930;20:443-80.

5. Prentice L, Stewart A. What is endosalpingiosis? Fertil Steril. 2012;98(4):942-47.

6. Zangmo R, Singh N, Kumar S, Vatsa R. Second look of endosalpingiosis: a rare entity. J Obstet Gynecol India. 2017;67:299.

7. Zapardiel I, Tobias-Gonzalez P, de Santiago J. Endosalpingiosis mimicking recurrent ovarian carcinoma. Taiwan J Obstet Gynecol. 2012;51:660e662.

8. Clement B, Young RH, Olsen NH, Hefferren JJ. Florid cystic endosalpingiosis with tumour-like manifestations: a report of four cases including the first reported cases of trans muralendosalpingiosis of the uterus. Ame J Surg Pathol. 1999;23:166-75.

9. Hemalatha AL, Ashok KP, Anoosha K, Indira CS. Cystic endosalpingiosis of uterine parametrium- a scarcely encountered and sparsely documented entity. J Clin Diagn Res. 2014;8(10):FD06-FD07.

10. Santeusanio G, Ventura L, Partenzi A, Spagnoli LG, Kraus FT. Omental endosalpingiosis with endometrial type stroma in a woman with extensive hemorrhagic pelvic endometriosis. Am J Clin Pathol. 1999;111:248-51.

Cite this article as: Shah A, Zangmo E, Jain K.

Endosalpingiosis with concurrent endometriosis of ovary masquerading as ovarian malignancy. Int $\mathrm{J}$ Reprod Contracept Obstet Gynecol 2020;9:2595-7. 